\title{
Strong Convergence for the Split Common Fixed-Point Problem for Total Quasi-Asymptotically Nonexpansive Mappings in Hilbert Space
}

\author{
Lawan Bulama Mohammed ${ }^{1}$ and A. Kılıçman ${ }^{2}$ \\ ${ }^{1}$ Department of Mathematics, University Putra Malaysia, 43400 Serdang, Selangor, Malaysia \\ ${ }^{2}$ Department of Mathematics and Institute for Mathematics Research, Universiti Putra Malaysia, 43400 Serdang, Selangor, Malaysia
}

Correspondence should be addressed to A. Kılıçman; akilic@upm.edu.my

Received 27 May 2014; Accepted 16 September 2014

Academic Editor: Salvador Romaguera

Copyright (C) 2015 L. B. Mohammed and A. Kılıçman. This is an open access article distributed under the Creative Commons Attribution License, which permits unrestricted use, distribution, and reproduction in any medium, provided the original work is properly cited.

\begin{abstract}
In this paper, we study and modify the algorithm of Kraikaew and Saejung for the class of total quasi-asymptotically nonexpansive case so that the strong convergence is guaranteed for the solution of split common fixed-point problems in Hilbert space. Moreover, we justify our result through an example. The results presented in this paper not only extend the result of Kraikaew and Saejung but also extend, improve, and generalize some existing results in the literature.
\end{abstract}

\section{Introduction}

Let $\langle\cdot, \cdot\rangle$ be an inner product space, $\|\cdot\|$ the corresponding norm, $E$ a Banach space, $H_{1}, H_{2}$ two Hilbert spaces, $A$ : $H_{1} \rightarrow H_{2}$ a bounded linear operator, and $A^{*}: H_{2} \rightarrow H_{1}$ an adjoint of $A$. Let $\left\{C_{i}\right\}_{i=1}^{p}$ and $\left\{Q_{j}\right\}_{j=1}^{r}$ be a nonempty, closed, convex subsets of $H_{1}$ and $H_{2}$, respectively.

A Banach space $E$ is said to satisfy Opial's condition (see [1]) if, for any sequence $\left\{x_{n}\right\}$ in $E, x_{n} \rightarrow x$ as $n \rightarrow \infty$ implies that

$$
\begin{array}{r}
\liminf _{n \rightarrow \infty}\left\|x_{n}-x\right\|<\liminf _{n \rightarrow \infty}\left\|x_{n}-y\right\|, \\
\forall y \in E, \quad y \neq x .
\end{array}
$$

And also, a Banach space $E$ is said to have Kadec-Klee property (see [1]), if, for any sequence $\left\{x_{n}\right\}$ in $E, x_{n} \rightarrow x$ and $\left\|x_{n}\right\| \rightarrow$ $\|x\|$ as $n \rightarrow \infty$ implies that

$$
x_{n} \longrightarrow x, \quad \text { as } n \longrightarrow \infty
$$

Remark 1. It is well known that each Hilbert space satisfied Opial and Kadec-Klee property.
The mapping $T: H \rightarrow H$ is said to be demiclosed at zero, if any sequence $\left\{x_{n}\right\}$ in $H$ there holds the following implication:

$$
x_{n} \rightarrow x, \quad T x_{n} \longrightarrow 0, \quad \text { as } n \longrightarrow \infty \Longrightarrow T x=0 .
$$

$T$ is said to be $\alpha$-strongly quasi-nonexpansive if there exists $\alpha>0$ with the property $\|T x-z\|^{2} \leq\|x-z\|^{2}-\alpha\|x-T x\|^{2}, \forall x \in$ $H$ and $z \in \operatorname{Fix}(T)$; this is equivalent to

$$
\begin{array}{r}
\langle x-z, T x-x\rangle \leq \frac{-1-\alpha}{2}\|x-T x\|^{2}, \\
\forall x \in H, \quad z \in \operatorname{Fix}(T) .
\end{array}
$$

$T$ is said to be quasi-nonexpansive, if $\operatorname{Fix}(T) \neq \emptyset$ such that $\|p-T x\| \leq\|p-x\|, \forall p \in \operatorname{Fix}(T)$ and $x \in H$, and $\left\{k_{n}\right\}-$ quasi-asymptotically nonexpansive mapping, if $\operatorname{Fix}(T) \neq \emptyset$ and there exists a sequence $\left\{k_{n}\right\} \subseteq[1, \infty)$ with $k_{n} \rightarrow 1$ such that, for each $n \geq 1,\left\|p-T^{n} x\right\|^{2} \leq k_{n}\|p-x\|^{2}, \forall p \in \operatorname{Fix}(T)$ and $x \in H$, and it is said to be $\left(\left\{v_{n}\right\},\left\{\mu_{n}\right\}, \xi\right)$-total quasiasymptotically nonexpansive mapping if $\operatorname{Fix}(T) \neq \emptyset$; and there exist nonnegative real sequences $\left\{v_{n}\right\},\left\{\mu_{n}\right\}$ in $[0, \infty)$ 
with $v_{n} \rightarrow 0$ and $\mu_{n} \rightarrow 0$ and a strictly continuous function $\xi: \mathfrak{R}^{+} \rightarrow \mathfrak{R}^{+}$with $\xi(0)=0$ such that, for each $n \geq 1$,

$$
\begin{array}{r}
\left\|p-T^{n} x\right\|^{2} \leq\|p-x\|^{2}+v_{n} \xi(\|p-x\|)+\mu_{n}, \\
\forall p \in \operatorname{Fix}(G), \quad x \in H .
\end{array}
$$

Remark 2. It is known that, the class of quasi-nonexpansive mapping contained in the class of $\left\{k_{n}\right\}$-quasi-asymptotically nonexpansive mapping and the class of $\left\{k_{n}\right\}$-quasi-asymptotically nonexpansive mapping is contained in the class of $\left(\left\{v_{n}\right\},\left\{\mu_{n}\right\}, \xi\right)$-total quasi-asymptotically nonexpansive mapping, see [2].

The mapping $T$ is said to be uniformly $L$-Lipschitzian if $\exists$ a constant $L>0$ such that, for each $n \geq 1,\left\|T^{n} x-T^{n} y\right\| \leq L \| x-$ $y \|, \forall x, y \in H$, and it is said to be semicompact, if, for any bounded sequence $x_{n} \subset H$ with $\lim _{n \rightarrow \infty}\left\|x_{n}-T x_{n}\right\|=0$, there exists subsequence $\left\{x_{n_{i}}\right\} \subset\left\{x_{n}\right\}$ such that $\left\{x_{n_{i}}\right\}$ converges strongly to some point $x^{*} \in H$.

The convex feasibility problems (CFP) are finding a vector $x^{*} \in H_{1}$ satisfying

$$
x^{*} \in \bigcap_{i=1}^{p} C_{i} .
$$

The problem of solving (6) has been intensively studied by numerous authors due to its various application in several physical problems such as approximation theorem, image recovery, signal processing, control theory, biomedical engineering, communication and geophysics (see [3-5]) and reference therein.

In 2005, Censor et al. (see [6]) introduced and studied the problem of multiple set split feasibility problems (MSSFP) which is formulated as finding a vector $x^{*} \in H_{1}$ with the property

$$
\begin{gathered}
x^{*} \in \bigcap_{i=1}^{p} C_{i}, \\
A x^{*} \in \bigcap_{j=1}^{r} Q_{j} .
\end{gathered}
$$

If, in (7), we take $p=r=1$, we get

$$
\begin{gathered}
x^{*} \in C, \\
A x^{*} \in Q .
\end{gathered}
$$

Equation (8) is known as the split feasibility problems (SFP) (see [7]), where $C$ and $Q$ are nonempty, closed, and convex subsets of $H_{1}$ and $H_{2}$, respectively. Since every closed convex subset of Hilbert space is the fixed-point set of its associating projection, then (6) and (7) become

$$
x^{*} \in \bigcap_{i=1}^{p} \operatorname{Fix}\left(U_{i}\right),
$$

$$
\begin{gathered}
x^{*} \in \bigcap_{i=1}^{p} \operatorname{Fix}\left(U_{i}\right), \\
A x^{*} \in \bigcap_{j=1}^{r} \operatorname{Fix}\left(T_{j}\right) .
\end{gathered}
$$

Equations (9) and (10) are called the common fixed-point problems (CFPP) and split common fixed-point problems (SCFPP), respectively, where $U_{i}: H_{1} \rightarrow H_{1}(i=$ $1,2,3, \ldots, p)$ and $T_{j}: H_{2} \rightarrow H_{2}(j=1,2,3, \ldots, r)$ are some nonlinear operators.

If we take $p=r=1$, problem (10) is reduced to find a point $x^{*} \in H_{1}$ with property

$$
\begin{aligned}
& x^{*} \in \operatorname{Fix}(U), \\
& A x^{*} \in \operatorname{Fix}(T) .
\end{aligned}
$$

Equation (11) is known as the two-set SCFPP.

In 2009, Censor and Segal [8] introduced the concept of SCFPP (10) in finite dimensional Hilbert space, who invented an algorithm for solving (11) which generate a sequence $\left\{x_{n}\right\}$ according to the following iterative procedure:

$$
x_{n+1}:=U\left(x_{n}+\gamma A^{*}(T-I) A x_{n}\right), \quad \forall n \geq 0,
$$

where the initial guess $x_{0} \in H$ is choosing arbitrarily and $0<\gamma<2 /\|A\|^{2}$.

In 2011, Moudafi [9] studied the convergence properties of relaxed algorithm for solving (10) for the class of quasinonexpansive operators $T$ such that $(I-T)$ is demiclosed at zero and he obtained the weak convergence results. Note that, in finite dimensional Hilbert space, weak and strong convergence are equivalent. Differently, in infinite dimensional cases, they are not the same. Moudafi's results guarantee only weak convergence results. In 2013, Mohammed $[10,11]$ utilized the strongly quasi-nonexpansive operators and quasinonexpansive operators to solve Moudafi's algorithm and he obtained weak and strong convergence results, respectively.

In 2014, Kraikaew and Saejung [12] also modified Moudafi's algorithm [9] and they obtained the strong convergent results as shown below.

Theorem 3 (see [12]). let $U: H_{1} \rightarrow H_{1}$ be a strongly quasinonexpansive operator and let T: $\mathrm{H}_{2} \rightarrow \mathrm{H}_{2}$ be a quasinonexpansive operator such that both $(I-U)$ and $(I-T)$ are demiclosed at zero. Let $A: H_{1} \rightarrow H_{2}$ be a bounded linear operator with $L=\left\|A A^{*}\right\|$. Suppose that $\Gamma \neq \emptyset$. Let $\left\{x_{n}\right\} \subset H_{1}$ be a sequence generated by

$$
\begin{gathered}
x_{0} \in H, \\
x_{n+1}=\alpha_{n} x_{0}+\left(1-\alpha_{n}\right) U\left(x_{n}+\gamma A^{*}(T-I) A x_{n}\right),
\end{gathered}
$$

where the parameters $\gamma$ and $\left\{\alpha_{n}\right\}$ satisfy the following conditions:

(a) $\gamma \in(0,1 / L)$;

(b) $\left\{\alpha_{n}\right\} \subset(0,1), \lim _{n \rightarrow \infty} \alpha_{n}=0$ and $\sum \alpha_{n}=\infty$. Then $x_{n} \rightarrow P_{\Gamma} x_{0}$. 
Motivated by these results, in this paper, we studied and modified the algorithm of Kraikaew and Saejung [12] for the class of total quasi-asymptotically nonexpansive mappings to solve the split common fixed-point problems (10) in the frame work of infinite dimensional Hilbert space. The results presented in this paper not only improve and extend some recent results of Kraikaew and Saejung [12], but also improve and extend some recent results of Censor and Segal [8], Moudafi [9], and Mohammed [10, 11] and many existing results.

Throughout this paper, we adopt the following notations.

(i) $I$ is the identity operator.

(ii) $\operatorname{Fix}(T)$ is the fixed-point set of $T$; that is, $\operatorname{Fix}(T)=\{x \in$ $H: T x=x\}$.

(iii) " $\rightarrow$ " and " $\rightarrow$ " denote the strong and weak convergence, respectively.

(iv) $\omega_{\omega}\left(x_{n}\right)$ denote the set of the cluster point of $\left\{x_{n}\right\}$ in the weak topology, that is, $\left\{\exists\left\{x_{n_{j}}\right\}\right.$ of $\left\{x_{n}\right\}$ such that $x_{n_{j}} \rightarrow$ $x\}$.

(v) $\Gamma$ is the solution set of split common fixed-point problems (10); that is,

$\Gamma$

$$
=\left\{x^{*} \in C=\bigcap_{i=1}^{p} \operatorname{Fix}\left(U_{i}\right), A x^{*} \in Q=\bigcap_{j=1}^{r} \operatorname{Fix}\left(T_{j}\right)\right\} .
$$

\section{Preliminaries}

In the sequel, we will make use of the following lemmas in proving our main results.

Lemma 4 (see [2]). Let $G: H \rightarrow H$ be a $\left(\left\{v_{n}\right\},\left\{\mu_{n}\right\}, \xi\right)$-total quasi-asymptotically nonexpansive mapping. Then for each $q \epsilon$ $\operatorname{Fix}(G)$ and $x \in H$, the following inequalities are equivalent: for each $n \geq 1$

$$
\begin{aligned}
& \left\|q-G^{n} x\right\|^{2} \leq\|q-x\|^{2}+v_{n} \xi(\|q-x\|)+\mu_{n} ; \\
& 2\left\langle x-G^{n} x, x-q\right\rangle \geq\left\|x-G^{n} x\right\|^{2}-v_{n} \xi(\|q-x\|) \\
& -\mu_{n} \text {; } \\
& 2\left\langle x-G^{n} x, q-G^{n}\right\rangle \leq\left\|x-G^{n} x\right\|^{2}+v_{n} \xi(\|q-x\|) \\
& +\mu_{n} \text {. }
\end{aligned}
$$

Lemma 5 (see [2]). Let $\left\{a_{n}\right\},\left\{b_{n}\right\},\left\{c_{n}\right\}$ be a sequences of nonnegative real numbers satisfying

$$
a_{n+1} \leq\left(1+c_{n}\right) a_{n}+b_{n} .
$$

If $\sum c_{n}<\infty$ and $\sum b_{n}<\infty$, then $\lim _{n \rightarrow \infty} a_{n}$ exists.

Lemma 6. Let $H$ be a Hilbert space, $C$ a nonempty closed convex subset of $H$, and $P_{C}$ a metric projection of $H$ onto $C$ satisfying $\left\langle x_{n}-x^{*}, x_{n}-P_{C} x_{n}\right\rangle \leq 0$, and then $\left\|P_{C} x_{n}-x_{n}\right\| \leq$ $\left\|P_{C} x_{n}-x^{*}\right\|, \forall n \geq 1$.
Proof. Let $x^{*} \in C$; then

$$
\begin{aligned}
&\left\|x_{n}-P_{C} x_{n}\right\|^{2}=\left\|x_{n}-x^{*}+x^{*}-P_{C} x_{n}\right\|^{2} \\
&=\left\|x_{n}-x^{*}\right\|^{2}+\left\|x^{*}-P_{C} x_{n}\right\|^{2} \\
&+2\left\langle x_{n}-x^{*}, x^{*}-P_{C} x_{n}\right\rangle \\
&=\left\|x_{n}-x^{*}\right\|^{2}+\left\|x^{*}-P_{C} x_{n}\right\|^{2} \\
&+2\left\langle x_{n}-x^{*}, x^{*}-x_{n}+x_{n}-P_{C} x_{n}\right\rangle \\
&=\left\|x_{n}-x^{*}\right\|^{2}+\left\|x^{*}-P_{C} x_{n}\right\|^{2} \\
&-2\left\|x_{n}-x^{*}\right\|^{2}+2\left\langle x_{n}-x^{*}, x_{n}-P_{C} x_{n}\right\rangle \\
&=\left\|x^{*}-P_{C} x_{n}\right\|^{2}-\left\|x_{n}-x^{*}\right\|^{2} \\
&+2\left\langle x_{n}-x^{*}, x_{n}-P_{C} x_{n}\right\rangle \\
& \leq\left\|x^{*}-P_{C} x_{n}\right\|^{2}, \\
& \Longrightarrow\left\|x_{n}-P_{C} x_{n}\right\| \leq\left\|x^{*}-P_{C} x_{n}\right\| .
\end{aligned}
$$

Lemma 7 (see [5]). If a sequence $\left\{x_{n}\right\}$ is Fejer monotone with respect to nonempty closed convex subset $C$, then the following hold:

(i) $x_{n} \rightarrow x^{*} \in C$ if and only if $\omega_{\omega} \subset C$;

(ii) the sequence $\left\{P_{C} x_{n}\right\}$ converges strongly to some point in $C$;

(iii) if $x_{n} \rightarrow x^{*} \in C$, then $x^{*}=\lim _{n \rightarrow \infty} P_{C} x_{n}$.

\section{Main Results}

Theorem 8. Let $H_{1}, H_{2}$ be two Hilbert spaces and $G: H_{1} \rightarrow$ $H_{1}, T: H_{2} \rightarrow H_{2}$ be $\left(\left\{v_{n_{1}}\right\},\left\{\mu_{n_{1}}\right\}, \xi_{1}\right),\left(\left\{v_{n_{2}}\right\},\left\{\mu_{n_{2}}\right\}, \xi_{2}\right)$-total quasi-asymptotically nonexpansive mappings and uniformly $L_{1}, L_{2}$-Lipschitzian continuous mappings such that $(I-G)$ and $(I-T)$ are both demiclosed at zero. Let $A: H_{1} \rightarrow H_{2}$ be a bounded linear operator and let $A^{*}: H_{2} \rightarrow H_{1}$ be an adjoint of $A$ with $L=\left\|A A^{*}\right\|$. Let $M$ and $M^{*}$ be positive constants such that $\xi(k) \leq \xi(M)+M^{*} k^{2}, \forall k \geq 0$. Assume that the solution set of SCFPP (14) is nonempty, and let $P_{\Gamma}$ be a metric projection of $H_{1}$ onto $\Gamma$ satisfying $\left\langle x_{n}-x^{*}, x_{n}-P_{\Gamma} x_{n}\right\rangle \leq 0$. Define the sequence $x_{n} \subset H_{1}$ by

$$
\begin{gathered}
x_{0} \in H_{1}, \\
u_{n}=\left(I+\gamma A^{*}\left(T^{n}-I\right) A\right) x_{n}, \\
x_{n+1}=\alpha_{n} u_{n}+\left(1-\alpha_{n}\right) G^{n} u_{n}, \quad \forall n \geq 1,
\end{gathered}
$$

where the parameter $\gamma, L,\left\{v_{n}\right\},\left\{\mu_{n}\right\},\left\{\xi_{n}\right\}$, and $\left\{\alpha_{n}\right\}$ satisfy the following conditions:

(a) $0<\alpha_{n}<1, \gamma \in(0,1 / L)$, where $L=\max \left\{L_{1}, L_{2}\right\}$; 
(b) $v_{n}=\max \left\{v_{n_{1}}, v_{n_{2}}\right\}, \mu_{n}=\max \left\{\mu_{n_{1}}, \mu_{n_{2}}\right\}$, and $\xi=$ $\max \left\{\xi_{1}, \xi_{2}\right\}$.

Then, the sequence $\left\{x_{n}\right\}$ defined by (18) converges strongly to $x^{*} \in \Gamma$.

Proof. To show that $x_{n} \rightarrow x^{*}$ as $n \rightarrow \infty$, it suffices to show $x_{n} \rightarrow x^{*}$ and $\left\|x_{n}\right\| \rightarrow\left\|x^{*}\right\|$ as $n \rightarrow \infty$.

The proof is divided into five steps as follows.

Step 1. In this step, we show that, for each $x^{*} \in \Gamma$, the following limit exists:

$$
\lim _{n \rightarrow \infty}\left\|x_{n}-x^{*}\right\|=\lim _{n \rightarrow \infty}\left\|u_{n}-x^{*}\right\|
$$

Let $x^{*} \in \Gamma$; this implies that $x^{*} \in C:=\bigcap_{i=1}^{p} \operatorname{Fix}\left(U_{i}\right)$ and $A x^{*} \in Q=\bigcap_{j=1}^{r} \operatorname{Fix}\left(T_{j}\right)$. From (18) and Lemma 4, we have

$$
\begin{aligned}
\| x_{n+1}- & x^{*} \|^{2} \\
= & \left\|\alpha_{n} u_{n}+\left(1-\alpha_{n}\right) G^{n} u_{n}-x^{*}\right\|^{2} \\
= & \alpha_{n}^{2}\left\|u_{n}-G^{n} u_{n}\right\|^{2} \\
& +2 \alpha_{n}\left\langle u_{n}-G^{n} u_{n}, G^{n} u_{n}-x^{*}\right\rangle+\left\|G^{n} u_{n}-x^{*}\right\|^{2} \\
= & \alpha_{n}^{2}\left\|u_{n}-G^{n} u_{n}\right\|^{2} \\
& +2 \alpha_{n}\left\langle u_{n}-x^{*}+x^{*}-G^{n} u_{n}, G^{n} u_{n}-x^{*}\right\rangle \\
& +\left\|G^{n} u_{n}-x^{*}\right\|^{2} \\
= & \alpha_{n}^{2}\left\|u_{n}-G^{n} u_{n}\right\|^{2}+2 \alpha_{n}\left\langle u_{n}-x^{*}, G^{n} u_{n}-x^{*}\right\rangle \\
& +\left(1-2 \alpha_{n}\right)\left\|G^{n} u_{n}-x^{*}\right\|^{2} \\
= & \alpha_{n}^{2}\left\|u_{n}-G^{n} u_{n}\right\|^{2}+2 \alpha_{n}\left\langle u_{n}-x^{*}, G^{n} u_{n}-u_{n}+u_{n}-x^{*}\right\rangle \\
& +\left(1-2 \alpha_{n}\right)\left\|G^{n} u_{n}-x^{*}\right\|^{2} \\
\leq & -\alpha_{n}\left(1-\alpha_{n}\right)\left\|u_{n}-G^{n} u_{n}\right\|^{2}+v_{n} \alpha_{n} \xi\left(\left\|u_{n}-x^{*}\right\|\right) \\
& +\mu_{n} \alpha_{n}+2 \alpha_{n}\left\|u_{n}-x^{*}\right\|^{2}+\left(1-2 \alpha_{n}\right) \\
& +\left(\left\|u_{n}-x^{*}\right\|^{2}+v_{n} \xi\left(\left\|u_{n}-x^{*}\right\|\right)+\mu_{n}\right) \\
= & -\alpha_{n}\left(1-\alpha_{n}\right)\left\|u_{n}-G^{n} u_{n}\right\|^{2}+\left(1-\alpha_{n}\right) v_{n} \xi \\
& +\left(\left\|u_{n}-x^{*}\right\|\right)+\left\|u_{n}-x^{*}\right\|^{2}+\left(1-\alpha_{n}\right) \mu_{n} \\
& -\alpha_{n}\left(1-\alpha_{n}\right)\left\|u_{n}-G^{n} u_{n}\right\|^{2}+\left(1+\left(1-\alpha_{n}\right) v_{n} M^{*}\right) \\
& +x^{*}+\left(1-\alpha_{n}\right)\left(v_{n} \xi(M)+\mu_{n}\right) . \\
& (1-10)
\end{aligned}
$$

On the other hand,

$$
\begin{aligned}
&\left\|u_{n}-x^{*}\right\|^{2} \\
&=\left\|x_{n}-x^{*}+\gamma A^{*}\left(T^{n}-I\right) A x_{n}\right\|^{2} \\
&=\left\|x_{n}-x^{*}\right\|^{2}+\gamma^{2}\left\|A^{*}\left(T^{n}-I\right) A x_{n}\right\|^{2} \\
&+2 \gamma\left\langle x_{n}-x^{*}, A^{*}\left(T^{n}-I\right) A x_{n}\right\rangle, \\
& \gamma^{2}\left\|A^{*}\left(T^{n}-I\right) A x_{n}\right\|^{2} \\
&=\gamma^{2}\left\langle A^{*}\left(T^{n}-I\right) A x_{n}, A^{*}\left(T^{n}-I\right) A x_{n}\right\rangle \\
&=\gamma^{2}\left\langle A A^{*}\left(T^{n}-I\right) A x_{n},\left(T^{n}-I\right) A x_{n}\right\rangle \\
& \leq \gamma^{2} L\left\|\left(T^{n}-I\right) A x_{n}\right\|^{2},
\end{aligned}
$$

by Lemma 4, it follows that

$$
\begin{aligned}
& 2 \gamma\left\langle x_{n}-x^{*}, A^{*}\left(T^{n}-I\right) A x_{n}\right\rangle \\
&= 2 \gamma\left\langle A x_{n}-A x^{*},\left(T^{n}-I\right) A x_{n}\right\rangle \\
& \leq-\gamma\left\|\left(T^{n}-I\right) A x_{n}\right\|^{2}+\gamma v_{n} M^{*} L\left\|x_{n}-x^{*}\right\|^{2} \\
&+\gamma\left(v_{n} \xi(M)+\mu_{n}\right) .
\end{aligned}
$$

By substituting (22) and (23) into (21), we obtained

$$
\begin{aligned}
\left\|u_{n}-x^{*}\right\|^{2} \leq & \left(1+\gamma v_{n} M^{*} L\right)\left\|x_{n}-x^{*}\right\|^{2}-\gamma(1-\gamma L) \\
& \cdot\left\|\left(T^{n}-I\right) A x_{n}\right\|^{2}+\gamma\left(v_{n} \xi(M)+\mu_{n}\right) .
\end{aligned}
$$

Substituting (24) into (20) and then simplifying, we have

$$
\begin{aligned}
\left\|x_{n+1}-x^{*}\right\|^{2} \leq & \left(1+\left(1-\alpha_{n}\right) v_{n} M^{*}\right) \\
& \cdot\left(\left(1+\gamma v_{n} M^{*} L\right)\left\|x_{n}-x^{*}\right\|^{2}\right. \\
& -\gamma(1-\gamma L)\left\|\left(T^{n}-I\right) A x_{n}\right\|^{2} \\
& \left.+\gamma\left(v_{n} \xi(M)+\mu_{n}\right)\right) \\
& -\alpha_{n}\left(1-\alpha_{n}\right)\left\|x_{n}-G^{n} u_{n}\right\|^{2} \\
& +\left(1-\alpha_{n}\right)\left(v_{n} \xi(M)+\mu_{n}\right) \\
\leq & \left(1+\left(1-\alpha_{n}\right) v_{n} M^{*}\right)\left(1+\gamma v_{n} M^{*} L\right) \\
& \cdot\left\|x_{n}-x^{*}\right\|^{2}-\gamma(1-\gamma L)\left\|\left(T^{n}-I\right) A x_{n}\right\|^{2} \\
& -\alpha_{n}\left(1-\alpha_{n}\right)\left\|x_{n}-G^{n} u_{n}\right\|^{2} \\
& +\left(1+\left(1-\alpha_{n}\right) v_{n} M^{*}\right) \gamma\left(v_{n} \xi(M)+\mu_{n}\right) \\
& +\left(1-\alpha_{n}\right)\left(v_{n} \xi(M)+\mu_{n}\right)
\end{aligned}
$$


and from (25), we deduce that

$$
\begin{aligned}
\| x_{n+1}- & x^{*} \|^{2} \\
\leq & \left(1+\gamma v_{n} M^{*} L+\left(1-\alpha_{n}\right) v_{n} M^{*}\left(1+\gamma v_{n} M^{*} L\right)\right) \\
& \cdot\left\|x_{n}-x^{*}\right\|^{2}+\left(1+\left(1-\alpha_{n}\right) v_{n} M^{*}\right) \\
& \cdot \gamma\left(v_{n} \xi(M)+\mu_{n}\right)+\left(1-\alpha_{n}\right)\left(v_{n} \xi(M)+\mu_{n}\right) .
\end{aligned}
$$

Therefore, from (26), we have

$$
\left\|x_{n+1}-x^{*}\right\|^{2} \leq\left(1+\beta_{n}\right)\left\|x_{n}-x^{*}\right\|^{2}+\eta_{n},
$$

where

$$
\begin{aligned}
\beta_{n}= & \gamma v_{n} M^{*} L+\left(1-\alpha_{n}\right) v_{n} M^{*}\left(1+\gamma v_{n} M^{*} L\right), \\
\eta_{n}= & \left(1+\left(1-\alpha_{n}\right) v_{n} M^{*}\right) \gamma\left(v_{n} \xi(M)+\mu_{n}\right) \\
& +\left(1-\alpha_{n}\right)\left(v_{n} \xi(M)+\mu_{n}\right) .
\end{aligned}
$$

Clearly, $\sum \beta_{n}<\infty$ and $\sum \eta_{n}<\infty$. Moreover, $\beta_{n} \rightarrow 0$ and $\eta_{n} \rightarrow 0$ as $n \rightarrow \infty$.

By Lemma 5, we conclude that $\lim _{n \rightarrow \infty}\left\|x_{n}-x^{*}\right\|$ exists.

We now prove that, for each $x^{*} \in \Gamma, \lim _{n \rightarrow \infty}\left\|u_{n}-x^{*}\right\|$ exists.

From (25), we deduce that

$$
\begin{aligned}
\gamma(1-\gamma L)\left\|\left(T^{n}-I\right) A x_{n}\right\|^{2} \leq & \left\|x_{n}-x^{*}\right\|^{2}-\left\|x_{n+1}-x^{*}\right\|^{2} \\
& +\beta_{n}\left\|x_{n}-x^{*}\right\|^{2}+\eta_{n} \\
\alpha_{n}\left(1-\alpha_{n}\right)\left\|u_{n}-G^{n} u_{n}\right\|^{2} \leq & \left\|x_{n}-x^{*}\right\|^{2}-\left\|x_{n+1}-x^{*}\right\|^{2} \\
& +\beta_{n}\left\|x_{n}-x^{*}\right\|^{2}+\eta_{n} .
\end{aligned}
$$

From (29), we deduce that

$$
\begin{aligned}
\lim _{n \rightarrow \infty}\left\|u_{n}-G^{n} u_{n}\right\| & =0, \\
\lim _{n \rightarrow \infty}\left\|A x_{n}-T^{n} A x_{n}\right\| & =0 .
\end{aligned}
$$

From (24), (30), and the fact that $\lim _{n \rightarrow \infty}\left\|x_{n}-x^{*}\right\|$ exists, then $\lim _{n \rightarrow \infty}\left\|u_{n}-x^{*}\right\|$ exists. Moreover, from (20), we deduce that

$$
\lim _{n \rightarrow \infty}\left\|u_{n}-x^{*}\right\|=\lim _{n \rightarrow \infty}\left\|x_{n}-x^{*}\right\|
$$

Step 2. In this step, we show that

$$
\begin{aligned}
& \lim _{n \rightarrow \infty}\left\|x_{n+1}-x_{n}\right\|=0, \\
& \lim _{n \rightarrow \infty}\left\|u_{n+1}-u_{n}\right\|=0 .
\end{aligned}
$$

Proof. It follows from (18) that

$$
\begin{aligned}
& \left\|x_{n+1}-x_{n}\right\| \\
& \quad=\left\|\alpha_{n} u_{n}+\left(1-\alpha_{n}\right) G^{n} u_{n}-x_{n}\right\| \\
& \quad=\left\|\left(1-\alpha_{n}\right)\left(G^{n} u_{n}-u_{n}\right)+u_{n}-x_{n}\right\| \\
& \quad=\left\|\left(1-\alpha_{n}\right)\left(G^{n} u_{n}-u_{n}\right)+A^{*}\left(T^{n}-I\right) A x_{n}\right\|,
\end{aligned}
$$

and in view of (30), we obtain that

$$
\lim _{n \rightarrow \infty}\left\|x_{n+1}-x_{n}\right\|=0 .
$$

Similarly, it follows from (30) and (34) that

$$
\begin{aligned}
\left\|u_{n+1}-u_{n}\right\|= & \|\left(I+\gamma A^{*}\left(T^{n+1}-I\right) A\right) x_{n+1} \\
& +\left(I+\gamma A^{*}\left(T^{n}-I\right) A\right) x_{n} \| \\
= & \| x_{n+1}-x_{n} \\
& +\gamma A^{*}\left(T^{n+1}-I\right) A x_{n+1}-\gamma A^{*}\left(T^{n}-I\right) A x_{n} \| \\
\longrightarrow & 0 \quad \text { as } n \longrightarrow \infty \\
\Longrightarrow & \lim _{n \rightarrow \infty}\left\|u_{n+1}-u_{n}\right\|=0 .
\end{aligned}
$$

Step 3. In this step, we show that

$$
\begin{array}{r}
\left\|u_{n}-G u_{n}\right\| \longrightarrow 0, \quad\left\|A x_{n}-T x_{n}\right\| \\
\text { as } n \longrightarrow 0,
\end{array}
$$

Proof. From the fact that $\left\|u_{n}-G^{n} u_{n}\right\| \rightarrow 0$ and $\left\|u_{n+1}-u_{n}\right\| \rightarrow$ 0 and $G$ is uniformly $L$-Lipschitzian continuous, it follows that

$$
\begin{aligned}
\left\|u_{n}-G u_{n}\right\| \leq & \left\|u_{n}-G^{n} u_{n}\right\|+\left\|G u_{n}-G^{n} u_{n}\right\| \\
\leq & \left\|u_{n}-G^{n} u_{n}\right\|+L\left\|u_{n}-G^{n-1} u_{n}\right\| \\
\leq & \left\|u_{n}-G^{n} u_{n}\right\|+L\left\|G^{n-1} u_{n}-G^{n-1} u_{n-1}\right\| \\
& \quad+L\left\|u_{n}-G^{n-1} u_{n-1}\right\| \\
\leq & \left\|u_{n}-G^{n} u_{n}\right\|+L^{2}\left\|u_{n}-u_{n-1}\right\| \\
& \quad+L\left\|u_{n}-u_{n-1}+u_{n-1}-G^{n-1} u_{n-1}\right\| \\
\leq & \left\|u_{n}-G^{n} u_{n}\right\|+L(L+1)\left\|u_{n}-u_{n-1}\right\| \\
& +L\left\|u_{n-1}-G^{n-1} u_{n-1}\right\| \longrightarrow \\
\Longrightarrow & \left\|u_{n}-G u_{n}\right\| \longrightarrow 0 .
\end{aligned}
$$

Similarly, from the fact that $\left\|A x_{n}-T^{n} A x_{n}\right\| \rightarrow 0, \| x_{n+1}-$ $x_{n} \| \rightarrow 0$, and $T$ is uniformly $L$-Lipschitzian continuous, it follows that $\left\|A x_{n}-T A x_{n}\right\| \rightarrow 0$.

Step 4. In this step, we show that

$$
x_{n} \rightarrow x^{*}, \quad u_{n} \rightarrow x^{*}, \quad \text { as } n \longrightarrow \infty .
$$

Proof. Since $\left\{u_{n}\right\}$ is bounded, then there exists a subsequence $u_{n_{i}} \subset u_{n}$ such that

$$
u_{n_{i}} \rightarrow x^{*}, \quad \text { as } i \longrightarrow \infty \text {. }
$$


From (39) and (36), we have

$$
\left\|u_{n_{i}}-G u_{n_{i}}\right\| \longrightarrow 0, \quad \text { as } i \longrightarrow \infty \text {. }
$$

From (39) and (40) and the fact that $(I-G)$ is demiclosed at zero, we get that $x^{*} \in \operatorname{Fix}(G)$.

Moreover, from (18), (39), and the fact $\left\|A x_{n}-T^{n} A x_{n}\right\| \rightarrow$ 0 , as $n \rightarrow \infty$, we have

$$
x_{n_{i}}=\alpha_{n} u_{n_{i}}-\gamma A^{*}\left(T^{n_{i}}-I\right) A x_{n_{i}} \longrightarrow x^{*} .
$$

By the definition of $A$, we get

$$
A x_{n_{i}} \rightarrow A x^{*}, \quad \text { as } i \longrightarrow \infty \text {. }
$$

In view of (36), we get

$$
\left\|A x_{n_{i}}-T A x_{n_{i}}\right\| \longrightarrow 0, \quad \text { as } i \longrightarrow \infty .
$$

From (42) and (43) and the fact that $(I-T)$ is demiclosed at zero, we have $A x^{*} \in \operatorname{Fix}(T)$, and this implies that $x^{*} \in \Gamma$.

Now, we show that $x^{*}$ is unique.

Suppose to the contrary that there exists another subsequence $u_{n_{j}} \subset u_{n}$ such that $u_{n_{j}} \rightarrow y^{*} \in \Gamma$ with $x^{*} \neq y^{*}$ by virtue of (19) and opial property of Hilbert space; we have

$$
\begin{aligned}
& \liminf _{i \rightarrow \infty}\left\|u_{n_{i}}-x^{*}\right\| \\
&< \liminf _{i \rightarrow \infty}\left\|u_{n_{i}}-y^{*}\right\|=\liminf _{n \rightarrow \infty}\left\|u_{n}-y^{*}\right\| \\
&= \liminf _{j \rightarrow \infty}\left\|u_{n_{j}}-y^{*}\right\|<\liminf _{j \rightarrow \infty}\left\|u_{n_{j}}-x^{*}\right\| \\
&=\liminf _{n \rightarrow \infty}\left\|u_{n}-x^{*}\right\|=\liminf _{i \rightarrow \infty}\left\|u_{n_{i}}-x^{*}\right\| \\
& \Longrightarrow \liminf _{i \rightarrow \infty}\left\|u_{n_{i}}-x^{*}\right\|<\liminf _{i \rightarrow \infty}\left\|u_{n_{i}}-x^{*}\right\|
\end{aligned}
$$

which is contradiction. Therefore $u_{n} \rightarrow x^{*}$. By using (18) and (30), we have

$$
x_{n}=u_{n}-\gamma A^{*}\left(T^{n}-I\right) A x_{n} \rightarrow x^{*}, \quad \text { as } n \longrightarrow \infty .
$$

Step 5. In this step, we show that

$$
\left\|x_{n}\right\| \longrightarrow\left\|x^{*}\right\|, \quad \text { as } n \longrightarrow \infty .
$$

To show (46), it suffices to show that $\left\|x_{n+1}\right\| \rightarrow\left\|x^{*}\right\|$ as $n \rightarrow$ $\infty$.

Proof. From Lemmas 6, 7, (27), and the fact that $\beta_{n} \rightarrow 0$ and $\eta_{n} \rightarrow 0$, we have

$$
\begin{aligned}
\left\|x_{n+1}\right\|-\left\|x^{*}\right\|^{2} \leq & \left\|x_{n+1}-x^{*}\right\|^{2} \\
\leq & \left(1+\beta_{n}\right)\left\|x_{n}-x^{*}\right\|^{2}+\eta_{n} \\
= & \left\|x_{n}-x^{*}\right\|^{2}+\beta_{n}\left\|x_{n}-x^{*}\right\|^{2}+\eta_{n} \\
= & \left\|x_{n}-P_{\Gamma} x_{n}+P_{\Gamma} x_{n}-x^{*}\right\|^{2} \\
& +\beta_{n}\left\|x_{n}-x^{*}\right\|^{2}+\eta_{n} \\
\leq & 4\left\|P_{\Gamma} x_{n}-x^{*}\right\|^{2}+\beta_{n}\left\|x_{n}-x^{*}\right\|^{2}+\eta_{n}
\end{aligned}
$$

$$
\begin{aligned}
\Longrightarrow \mid\left\|x_{n+1}\right\|-\left\|x^{*}\right\|^{2} \leq 4 & \left\|P_{\Gamma} x_{n}-x^{*}\right\|^{2} \\
& +\beta_{n}\left\|x_{n}-x^{*}\right\|^{2}+\eta_{n} \\
\Longrightarrow \lim _{n \rightarrow \infty} \mid\left\|x_{n+1}\right\|-\left\|x^{*}\right\|^{2} \leq & 4 \lim _{n \rightarrow \infty}\left\|P_{\Gamma} x_{n}-x^{*}\right\|^{2} \\
& +\lim _{n \rightarrow \infty} \beta_{n}\left\|x_{n}-x^{*}\right\|^{2} \\
& +\lim _{n \rightarrow \infty}\left(\eta_{n}\right) \\
\Longrightarrow \lim _{n \rightarrow \infty}\left|\left\|x_{n+1}\right\|-\left\|x^{*}\right\|\right|^{2}= & 0 .
\end{aligned}
$$

From (38) and (46), we conclude that $x_{n} \rightarrow x^{*}$, as $n \rightarrow$ $\infty$.

Corollary 9. Let $H_{1}, H_{2} A$, and $A^{*}$, be as in Theorem 8. Let $G$ and $T$ be $\left\{k_{n_{1}}\right\},\left(\left\{k_{n_{2}}\right\}\right)$ quasi-asymptotically nonexpansive and uniformly $L_{1}, L_{2}$-Lipschitzians continuous mappings such that $(I-G)$ and $(I-T)$ are both demiclosed at zero. Let $L=\left\|A A^{*}\right\|$, and let $M$ and $M^{*}$ be constants such that $\xi(k) \leq$ $\xi(M)+M^{*} k^{2}, \forall k \geq 0$. Assume that the solution set of SCFPP (14) is nonempty, and let $P_{\Gamma}$ be a metric projection of $H_{1}$ onto $\Gamma$ satisfying $\left\langle x_{n}-x^{*}, x_{n}-P_{\Gamma} x_{n}\right\rangle \leq 0$. Let the sequence $\left\{x_{n}\right\}$ be defined as in Theorem 8 where the parameters $\alpha_{n}, \gamma,\left\{k_{n}\right\}$, and $L$ satisfy the following conditions:

(a) $\alpha_{n} \subset(0,1), \gamma \in(0,1 / L)$, where $L=\max \left\{L_{1}, L_{2}\right\}$;

(b) $k_{n}=\max \left\{k_{n_{1}}, k_{n_{2}}\right\}$.

Then the sequence $\left\{x_{n}\right\}$ defined as in Theorem 8 converges strongly to $x^{*} \in \Gamma$.

Proof. By Remark $2 G$ and $T$ are $\left(\left\{v_{n}\right\},\left\{\mu_{n}\right\}, \xi\right)$-total quasiasymptotically nonexpansive mappings with $\left\{v_{n}\right\}=\left\{k_{n}-1\right\}$, $\mu_{n}=0$, and $\xi(k)=k^{2}, \forall k \geq 0$. Therefore, all the conditions in Theorem 8 are satisfied. The conclusions of this corollary follow directly from Theorem 8 .

Corollary 10. Let $H_{1}, H_{2} A$, and $A^{*}$ be as in Theorem 8. Let $G$ and $T$ be two quasi-nonexpansive and uniformly $L_{1}, L_{2}$ Lipschitzian continuous mappings such that $(I-G)$ and $(I-T)$ are both demiclosed at zero. Let $L=\left\|A A^{*}\right\|$, and let $M$ and $M^{*}$ be positive constants such that $\xi(k) \leq \xi(M)+M^{*} k^{2}, \forall k \geq 0$. Assume that the solution set of SCFPP (14) is nonempty, and let $P_{\Gamma}$ be a metric projection of $H$ onto $\Gamma$ satisfying $\left\langle x_{n}-\right.$ $\left.x^{*}, x_{n}-P_{\Gamma} x_{n}\right\rangle \leq 0$. Let the sequence $\left\{x_{n}\right\}$ be defined as in Theorem 8 where the parameters $\left\{\alpha_{n}\right\}, \gamma$, and L, satisfy the following conditions:

(a) $\left\{\alpha_{n}\right\} \subset(0,1), \gamma \in(0,1 / L)$, where $L=\max \left\{L_{1}, L_{2}\right\}$.

Then, the sequence $\left\{x_{n}\right\}$ defined as in (18) converges strongly to $x^{*} \in \Gamma$.

Proof. By Remark $2 G$ and $T$ are $(\{1\})$-quasi-asymptotically nonexpansive mappings. Therefore, all the conditions in Corollary 9 are satisfied. The conclusions of this corollary follow directly from Corollary 9. 
Now we give an example of our theorem.

Example 11. Let $B$ be a unit ball in a real Hilbert space $l_{2}$, and let $T: B \rightarrow B$ be a mapping define by

$$
\begin{array}{r}
T:\left(x_{1}, x_{2}, x_{3}, \ldots\right) \longrightarrow\left(0, x_{1}^{2}, a_{2} x_{2}, a_{3} x_{3}, \ldots\right), \\
\left(x_{1}, x_{2}, x_{3}, \ldots\right) \in B,
\end{array}
$$

where $\left\{a_{i}\right\}$ is a sequence in $(0,1)$ such that $\prod_{i=2}^{\infty}\left(a_{i}\right)=1 / 2$.

It is proved in Goebel and Kirk [13] that

(a) $\|T x-T y\| \leq 2\|x-y\|$,

(b) $\left\|T^{n} x-T^{n} y\right\| \leq 2 \prod_{i=2}^{n}\left(a_{i}\right)\|x-y\| \forall x, y \in B$ and $n \geq 2$. Let $k_{1}^{1 / 2}=2$ such that $k_{n}^{1 / 2}=2 \prod_{i=2}^{n}\left(a_{i}\right)$, for $n \geq 2$; then

$$
\lim _{n \rightarrow \infty} k_{n}=\lim _{n \rightarrow \infty}\left(2 \prod_{i=2}^{n} a_{i}\right)=1 .
$$

Let $v_{n}=k_{n}-1, \forall n \geq 1$, let $\xi(t)=t^{2}, \forall t \geq 0$, let and $\left\{\mu_{n}\right\}$ be a nonnegative real sequence such that $\mu_{n} \rightarrow \infty$ as $n \rightarrow \infty$. From (a), (b) and $\forall x, y \in B$ and $n \geq 1$, we have

$$
\left\|T^{n} x-T^{n} y\right\|^{2} \leq\|x-y\|^{2}+v_{n}\|x-y\|^{2}+\mu_{n} .
$$

Again, since $0 \in B$ and $0 \in \operatorname{Fix}(T)$, this implies that $\operatorname{Fix}(T) \neq$ $\emptyset$. From the above equation, we have

$$
\left\|p-T^{n} y\right\|^{2} \leq\|p-y\|^{2}+v_{n} \xi(\|p-y\|)+\mu_{n} .
$$

This show that $T$ is total quasi-asymptotically nonexpansive mapping.

Example 12. Let $H_{1}=H_{2}=l_{2}$ be a real Hilbert spaces, $C, Q$ two unit balls in $l_{2}$, and $T: C \rightarrow C, G: Q \rightarrow Q$ two mappings defined by

$$
\begin{array}{r}
T:\left(x_{1}, x_{2}, x_{3}, \ldots\right) \longrightarrow\left(0, x_{1}^{2}, a_{2} x_{2}, a_{3} x_{3}, \ldots\right), \\
\left(x_{1}, x_{2}, x_{3}, \ldots\right) \in C, \\
G:\left(y_{1}, y_{2}, y_{3}, \ldots\right) \longrightarrow\left(0, y_{1}^{2}, b_{2} y_{2}, b_{3} y_{3}, \ldots\right), \\
\left(y_{1}, y_{2}, y_{3}, \ldots\right) \in Q
\end{array}
$$

such that $(I-T)$ and $(I-G)$ are both demiclosed at zero, where $\left\{a_{i}\right\}$ and $\left\{b_{i}\right\}$ are sequences in $(0,1)$ such that $\prod_{i=2}^{\infty}\left(a_{i}\right)=$ $\prod_{i=2}^{\infty}\left(b_{i}\right)=1 / 2$. Let $A, A^{*}, M, M^{*} \xi(k)$, and $P_{\Gamma}$ be as in Theorem 8 . And assume that conditions (a)-(b) in Theorem 8 are satisfied. Then, the sequence $\left\{x_{n}\right\}$ defined in Theorem 8 converges strongly to $x^{*} \in \Gamma$.

Proof. By Example 11, it follows that $G$ and $T$ are both $\left(\left\{v_{n}\right\},\left\{\mu_{n}\right\}, \xi\right)$-total quasi-asymptotically nonexpansive mappings; moreover from Example 11 (b) we have that $G$ and $T$ are both uniformly $L_{1}, L_{2}$-Lipschitzian with $L_{1}=2 \prod_{i=2}^{n}\left(a_{i}\right)$ and $L_{2}=2 \prod_{i=2}^{n}\left(b_{i}\right)$; also by our hypothesis $(I-G)$ and $(I-T)$ are both demiclosed at zero. Therefore, all the conditions in Theorem 8 are satisfied. Hence, the conclusions of this corollary follow directly from Theorem 8 .

\section{Conflict of Interests}

The authors declare that there is no conflict of interests regarding the publication of this paper.

\section{Acknowledgment}

The authors gratefully acknowledge that this research was partially supported by the University Putra Malaysia under GP-IBT Grant Scheme having project number GP-IBT/2013/ 9420100 .

\section{References}

[1] Z. Opial, "Weak convergence of the sequence of successive approximations for nonexpansive mappings," Bulletin of the American Mathematical Society, vol. 73, pp. 591-597, 1967.

[2] X. R. Wang, S.-S. Chang, L. Wang, and Y.-H. Zhao, "Split feasibility problems for total quasi-asymptotically nonexpansive mappings," Fixed Point Theory and Applications, vol. 2012, article 151, 2012.

[3] H. Stark, Ed., Image Recorvery Theorem and Application, Academic Press, Orlando, Fla, USA, 1987.

[4] P. L. Combettes, "The convex feasibility problem in image recovery," in Advances in Imaging and Electron Physics, P. Hawkes, Ed., vol. 95, pp. 155-270, Academic Press, New York, NY, USA, 1996.

[5] H. H. Bauschke and J. M. Borwein, "On projection algorithms for solving convex feasibility problems," SIAM Review, vol. 38, no. 3, pp. 367-426, 1996.

[6] Y. Censor, T. Elfving, N. Kopf, and T. Bortfeld, "The multiplesets split feasibility problem and its applications for inverse problems," Inverse Problems, vol. 21, no. 6, pp. 2071-2084, 2005.

[7] Y. Censor and T. Elfving, "A multiprojection algorithm using Bregman projections in a product space," Numerical Algorithms, vol. 8, no. 2-4, pp. 221-239, 1994.

[8] Y. Censor and A. Segal, "The split common fixed point problem for directed operators," Journal of Convex Analysis, vol. 16, no. 2, pp. 587-600, 2009.

[9] A. Moudafi, "A note on the split common fixed-point problem for quasi-nonexpansive operators," Nonlinear Analysis: Theory, Methods \& Applications, vol. 74, no. 12, pp. 4083-4087, 2011.

[10] L. B. Mohammed, "A note on the split common fixed-point problem for strongly quasinonexpansive operator in Hilbert space," International Journal of Innovative Research and Studies, vol. 2, no. 8, pp. 424-434, 2013.

[11] L. B. Mohammed, "Strong convergence of an algorithm about quasi-nonexpansive mappings for the split common fixed-pint problem in Hilbert space," International Journal of Innovative Research and Studies, vol. 2, no. 8, pp. 298-306, 2013.

[12] R. Kraikaew and S. Saejung, "On split common fixed point problems," Journal of Mathematical Analysis and Applications, vol. 415, no. 2, pp. 513-524, 2014.

[13] K. Goebel and W. A. Kirk, "A fixed point theorem for asymptotically nonexpansive mappings," Proceedings of the American Mathematical Society, vol. 35, pp. 171-174, 1972. 


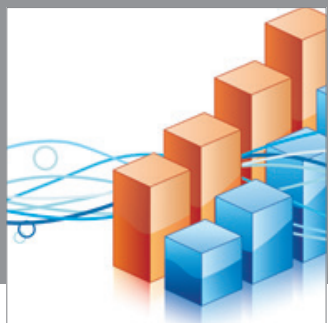

Advances in

Operations Research

mansans

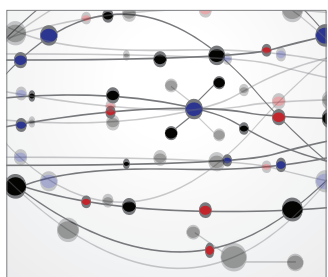

The Scientific World Journal
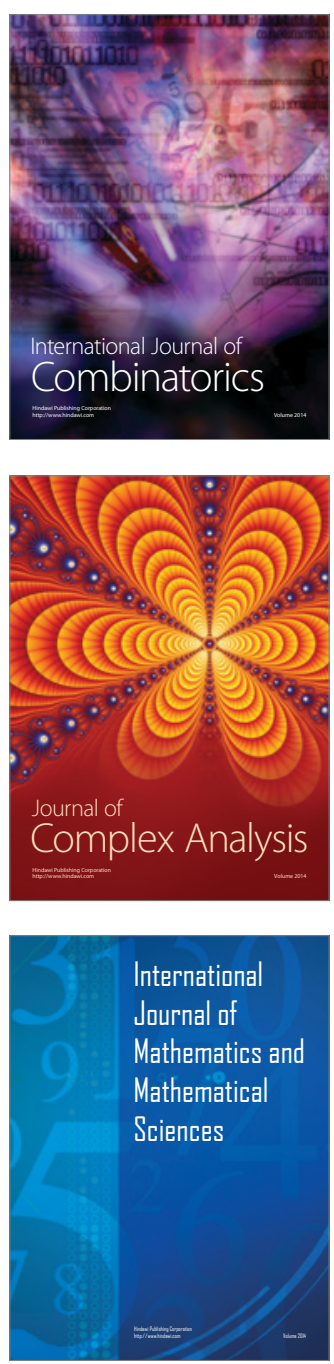
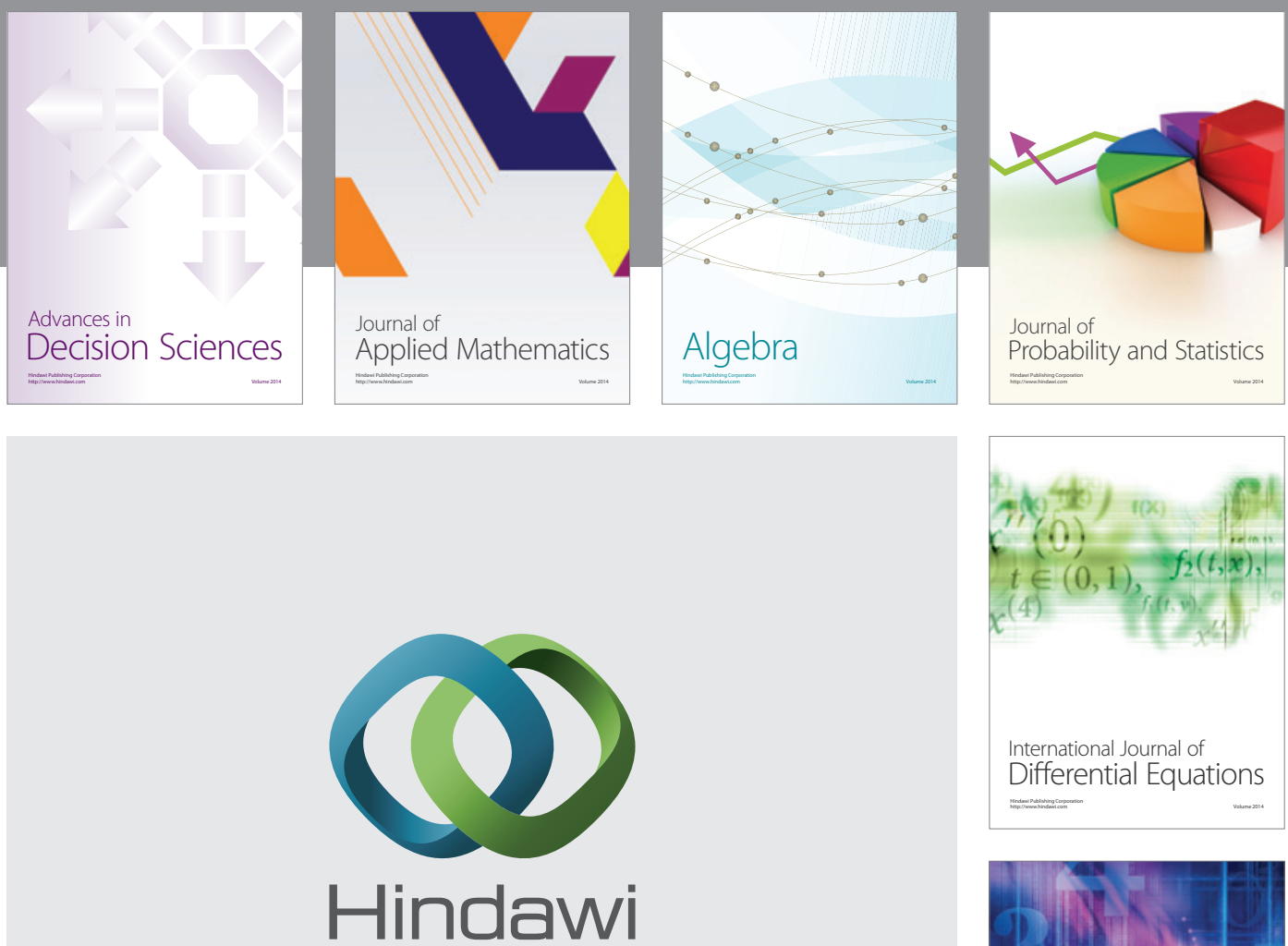

Submit your manuscripts at http://www.hindawi.com
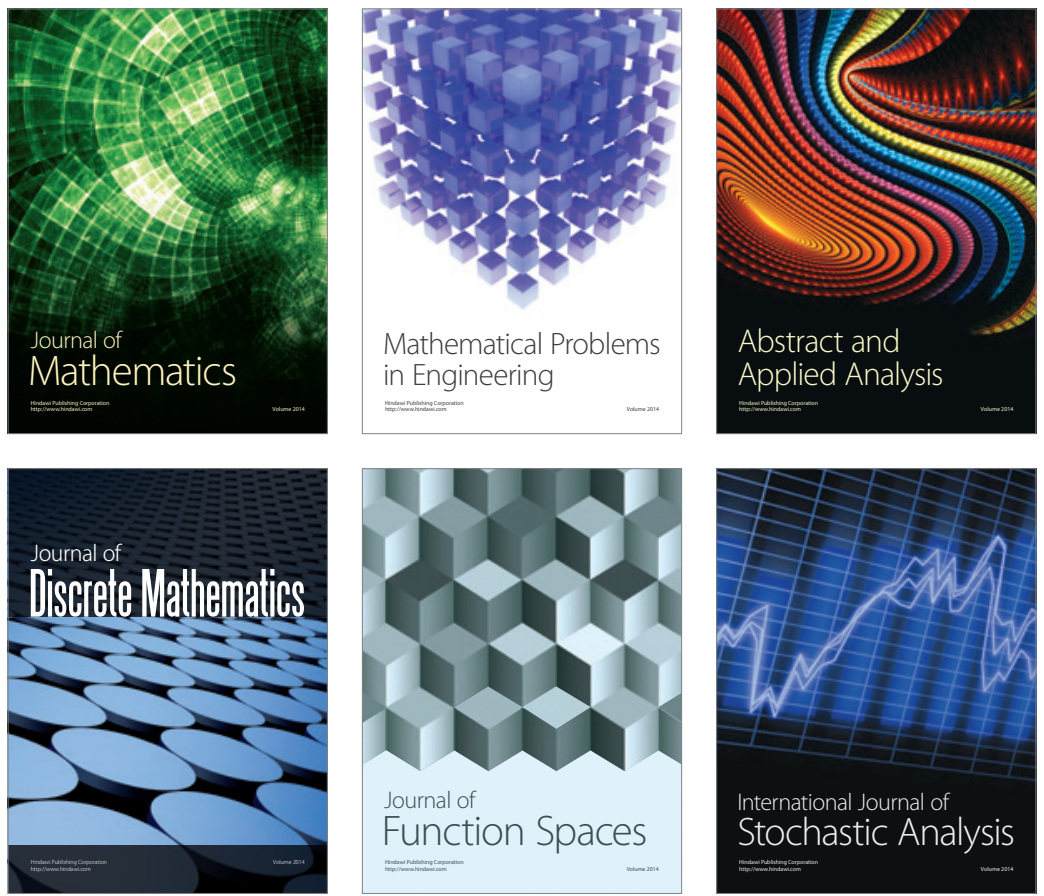

Journal of

Function Spaces

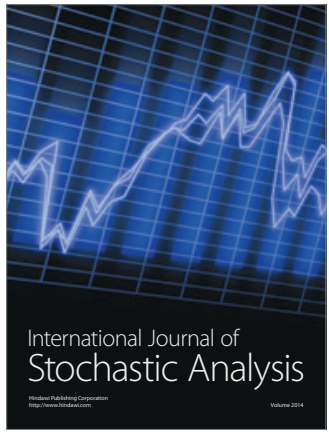

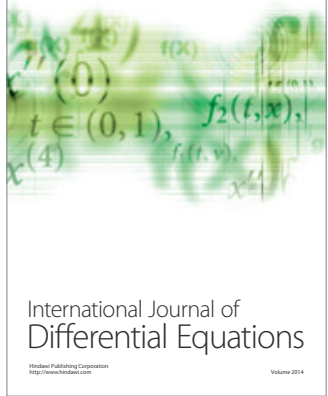
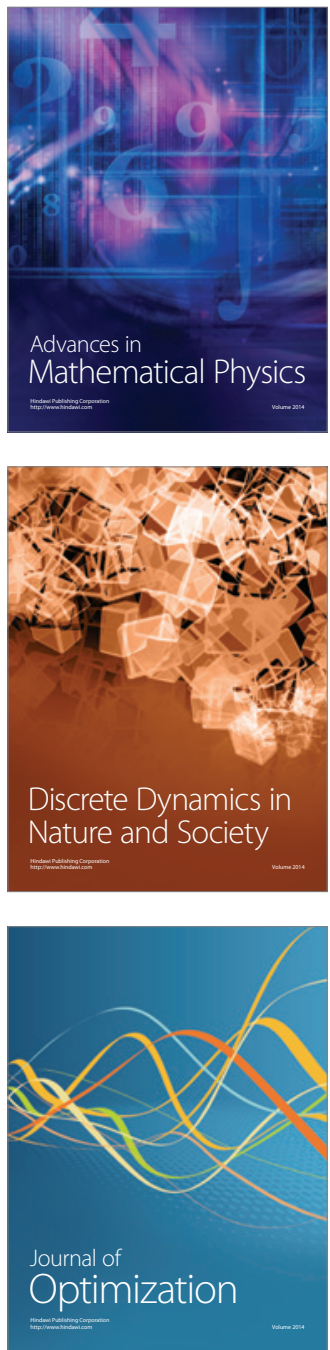